\title{
Reflection and Discussion on "One-Stop" Service Mode of Chinese Universities in the context of COVID-19 -- Based on the Perspective of Student Affairs
}

\author{
Yang Zhen ${ }^{1, \mathrm{a}}$, Liu Rongshan ${ }^{2, \mathrm{~b}^{*}}$, Sun Gaoang ${ }^{3, \mathrm{c}^{* *}}$, Chen Ying ${ }^{4, \mathrm{~d}^{* * *}}$ \\ ${ }^{1}$ Student Affairs office of Guangdong Ocean University, Huguang Town, Mazhang District, Zhanjiang, China \\ ${ }^{2}$ Department of Sociology, School of Law and Political Science, Guangdong Ocean University, Huguang Town, \\ Mazhang District, Zhanjiang, China \\ ${ }^{3}$ Department of Public Administration, School of Management, Guangdong Ocean University, Huguang Town, \\ Mazhang District, Zhanjiang, Guangdong, China \\ ${ }^{4}$ Department of Financial Management, School of Management, Guangdong Ocean University, Huguang Town, \\ Mazhang District, Zhanjiang, Guangdong, China \\ ayangz@gdou.edu.cn \\ b*liurongshan@stu.gdou.edu.cn \\ $c^{* * *}$ sungaoang@stu.gdou.edu.cn \\ $d^{* * * *} c_{-} 123 @$ stu.gdou.edu.cn
}

\begin{abstract}
With the continuous deepening of China's higher education reform and the epidemic of COVID-19 in 2020, Chinese colleges and universities have entered a critical period in the field of student work in the construction of "one-stop" service mode, and the demand for "one-stop" service from faculty, staff and students in their colleges and universities is increasingly deepening. Vigorously promoting China's colleges and universities "one-stop" service mode, can effectively solve a large number of "student affairs" produced by the process of China's high education from "elite education" to "mass education", which helps to increase work efficiency of college students, to form resultant force of organization structure, give full play and make good use of college students job responsibilities of each position, so that the academic level in conjunction with the scientific and reasonable student work to further improve. This paper integrated use of a variety of research methods in the process of research, on the basis of the basic definition, according to the actual situation, based on the perspective of student affairs, reflection and summary of present Chinese universities in the novel coronavirus outbreak under the big background of the problems existing in the "one-stop" service model and the reasons, and put forward a practical optimization approaches, including promoting the "one-stop" service model combined with "Internet" and other countermeasures.
\end{abstract}

Keywords: "One-stop" Service Model, Chinese Universities, Student Affairs, COVID-19

\section{新冠疫情背景下中国高校 “一站式”服务模式反思 \\ 与探讨一一基于学生事务视角}

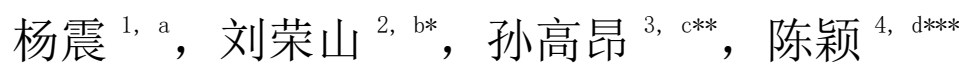

'广东海洋大学学生处, 湖光镇, 麻章区, 湛江市, 中国

2 广东海洋大学法政学院社会学系, 湖光镇, 麻章区, 湛江市, 中国

3 广东海洋大学管理学院公共管理系, 湖光镇, 麻章区, 湛江市, 中国

4 广东海洋大学管理学院财务管理系, 湖光镇, 麻章区, 湛江市, 中国 


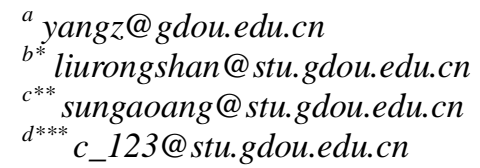

\section{摘要}

随着中国高等教育改革的持续深化，在 2020 年新冠疫情肆虐的背景下，中国各高校在建设 “一 站式” 服务模式的学生工作领域进入了关键时期, 学校教职工和学生对所属高校的 “一站式” 服务的需求日益加深。大力推进中国高校 “一站式” 服务模式，能够有效解决在中国高等教育 从 “精英教育” 跨向 “大众教育” 之际大量产生的 “学生事务” , 进而有助于提升高校处理学 生事务能力, 形成合理的组织结构, 充分发挥与利用学生工作中心作用, 从而使高校的学术水 平在科学合理的 “一站式” 服务中心配合下得以进一步提升。因此本论文在开展研究过程中综 合运用多种研究方法, 在基础概念界定的基础上，根据各高校 “一站式” 服务中心的实际情况， 基于学生事务的视角, 反思与总结现阶段中国高校在新冠疫情的大背景下 “一站式” 服务模式 存在的问题及其原因，并提出具有可实施性的优化途径，其中包括促进 “一站式” 服务模式与 “互联网”相结合等对策。

关键词: “一站式”服务模式，中国高校，学生事务，新冠疫情

\section{1. 问题的提出}

随着中国高等教育改革不断深化, 中国各地区、 各类高校逐渐重视并着手探讨高效率、高质量解决 学生事务的具体途径; 同时高校增加招收本科生、 研究生名额, 从而高等教育逐步从 “精英教育” 迈 向 “大众教育”, 大学生数量的快速增长加大了 “学 生事务” 工作量, 导致 “学生事务” 成为了高校函 待解决的现实问题。

目前, 大多数高校在学生管理模式、学生服务模 式、学生教学模式仍执行传统的模式, 这类传统模式 逐渐显露出各种现实困境。不难理解, 高等教育不断 改革、不断创新, 就是要确保高等教育在制度上能与 时俱进, 在执行上能迎刃而解。历年来, 部分高校、 学者开始探讨研究、创新发展高校学生管理与服务制 度, 从多方面探讨与解决学生事务这一迫切问题。

在探讨高校管理与服务的模式上，部分高校行 政职工、学者参考国内外高校处理学生、教职工事 务的创新模式, 根据高校现实情况, 逐步发展出本 土化的管理与服务模式一一 “一站式” 服务模式。 2020 年全球爆发了新型冠状病毒肺炎, 对社会造成 了严重的影响, 同时其传染性及潜伏性对高校学生 管理部门及学生服务部门提出了更高的要求, 所以 现阶段高校 “一站式” 服务模式必须进行深度反思 与探讨, 确保 “一站式”服务模式能够做到高效化、 秩序化、规范化服务学生, 同时应当发展与完善在 “突发事件” 中仍能发挥作用的 “一站式” 服务模 式。本文主要从 “学生事务” 角度探讨新冠肺炎疫 情影响下高校 “一站式” 服务的创新与发展方向。

\section{2. “一站式” 服务模式具体特点}

\section{1. 服务性}

“一站式” 服务模式是以学生、教职工为服务 对象, 同时兼具管理职能。学生的 “一站式” 模式 基于传统模式的 “管理” 与 “处理” 这类职能逐步 转变为 “服务”与 “管理”, 逐渐淡化 “管理” 理 念, 从 “行政部门的解决能力” 为核心转向 “学生 的事务需求” 为核心, 即坚持 “学生为本” 工作理 念, 发挥好学生主体性作用, 从学生事务需求出发, 把 “学生” 类比为 “顾客” 更好地解决学生事务。

\section{2. 综合性}

“一站式” 服务模式是综合高校多个行政部门 或组织的统一体, 汇聚学生处、教务处、后勤部、 团委等部门形成 “一站式” 服务的学生工作中心。 将各类行政部门汇聚起来, 能极大程度地提高办事 效率，推动发展 “只进一扇门”、“最多跑一次” 新 形态。同时各部门形成具备综合性的 “一站式” 服 务中心能有效避免 “踢皮球” 现象, 确保学生事务 能及时得到处理与解决。 


\section{3. 多样性}

“一站式” 服务模式从部门综合性上决定了其 服务多样性，即综合性的“一站式” 服务中心能满 足各类学生事务需求, 如新生入学需办理相关后勤 事务、以及毕业生办理就业事务、档案事务等等。 从新生入学到毕业生离校都能通过 “一站式”服务 模式统一完成。“一站式” 服务模式的多样性特征 具体表现为服务直接性与服务便利性。

\section{4. 专业性}

“一站式”服务模式的创新特点体现于其 “专 业性”，传统模式容易出现职工人员冗余以及各行 政部门工作量与工作压力有所差异，甚至部分岗位 就是 “闲职”。所以 “一站式” 服务模式在综合多 部门后, 可进行职位调整, 根据学生事务需求, 配 备人员, 形成合理的组织结构, 充分发挥好各个岗 位的职能, 从 “需求” 角度和 “供给” 角度推进高 校职工结构改革一一将合适的职工放在合适的岗 位, 将合适的数量配备在合适的部门, 这充分彰显 了 “一站式” 服务模式的专业性特点。

\section{5. 高效性}

高校推进“一站式”服务模式, 从根本上来说， 就是发挥好高校行政部门职能，即提升各行政部门 解决学生事务能力。“一站式” 服务模式一方面解 决了学生在办理事务过程中的行程问题（地理问 题), 另一方面 “一站式” 服务模式在组织结构上 其属于同一组织、同一部门，即 “一站式” 服务模 式能有效解决传统模式中各部门衔接问题, 提高各 部门工作效率与服务水平。

\section{3. “一站式” 服务模式现实困境}

“一站式” 服务模式是高校管理与服务模式的 创新成果, 其核心是聚多部门于一体, 从过去各部 门单一性职能演变成 “一站式” 服务的综合性职能。 经过国内部分高校的实际实践, 不难发现 “一站式” 服务模式仍需解决部分关键问题, 特别是在新冠肺 炎疫情影响下，“一站式” 服务暴露了部分潜在问 题。“一站式” 服务模式现实困境如下:

\section{1. 综合各部门止于表面}

“一站式” 服务中心是多个行政部门的统一体， 从“学生需求” 角度出发, “一站式” 服务模式综 合性特征能满足 “学生需求” 多样性的需求。但部 门高校 “一站式” 中心容易出现各行政部门仅进行
机械化统一，即 “一站式” 服务中心只完成地理位 置的统一, 其实际职能在配合与传递过程中仍保持 着 “各自为战” 状态, 这无疑违背 “一站式” 服务 模式综合性特征, 亦剪断了解决学生事务的衔接链。

\section{2. 信息化技术亟需加强}

“互联网+”发展模式是现阶段我国社会发展 的重要途径, 如 “互联网+医疗”、“互联网+农业” 等等。但目前极大部分高校其信息化应用水平仍处 于较低水平甚至极低水平, 信息化应用技术仅停滞 在 “图书馆搜索” “后勤充值服务” 等基础性服务。 低水平的信息化应用技术将降低 “一站式” 服务中 心工作效率, 一方面影响了学生在处理自身事务上 的时间规划, 另一方面是增加 “一站式” 服务中心 职工人员工作压力的 “隐形途径”。在疫情影响下, 高水平信息化技术能够更有效阻止疫情传播, 即高 水平信息化技术在处理学生事务过程中能兼顾学 生生命健康安全与高效率完成学生事务。

\section{3. 职工人员综合能力较弱, 职工人员结 构有待优化}

部分高校行政职工在处理和解决学生事务过 程中, 容易出现 “官僚主义” “消极急工” 等负面 现象。上述现象直接反映出部分职工人员需提高自 身价值观念水平。除此, 部分高校 “一站式” 服务 中心职工人员的人员结构缺乏科学性与合理性, 特 别是职工年龄结构问题和专业结构问题: 如职工年 龄结构过于年轻化或过于老龄化都容易导致办事 效率低下, 工年龄结构过于老龄化, 其职工能力难 以满足信息技术操作要求等等。

\section{4. “一站式” 服务模式优化途径}

\section{1. 促进“一站式” 服务模式与 “互联网 + ” 相结合}

在学生管理与学生服务过程中, “一站式” 服 务中心模式基本以线下形式为主, 但各高校应重视 并完善解决学生事务的线上途径, 充分发挥信息化 技术作用, 将 “互联网+服务” 作为 “一站式” 服 务模式的重要环节。在新冠疫情影响下, “互联网+ 服务” 模式更符合防疫控疫实际要求, 能有效推动 打赢打好防疫阻击战。“互联网+服务” 服务模式的 构建可通过微信公众号、“一站式” 服务中心网站 以及 APP 等现代信息技术完成。从 “互联网+” 途 径对 “一站式” 服务模式进行丰富与创新, 既是满 足防疫控疫的实际举措, 又是构建高水平学生管理 与服务平台的优化表现。 


\section{2. 创新改革 “一站式” 服务中心组织结 构}

“一站式” 服务由多部门聚集、演变而成, 从 功能性角度思考, 高校领导部门必须从根本上、制 度上确保 “一站式” 服务中心是一个具备服务性、 综合性、高效性、多样性、专业性特点的职能组织。 那么各高校应将 “一站式” 服务中心进行规范化、 合法化的聚集与调整, 促进各部门有机融合、各事 务有效衔接, 而并非各部门机械化地统一。同时高 校领导部门需进一步完善 “一站式”服务中心的监 督管理机制, 确保 “一站式” 服务模式高效、稳定 运行。

\section{3. 调整“一站式”服务中心人员及优化 职工技术技能培训}

“一站式” 服务中心是高校处理学生事务的创 新变项。调整 “一站式” 服务中心人员以及开展技 术技能培训必须精准定位 “学生事务需求”。具体 而言，可通过调查了解各类学生事务的实际情况， 如 “学生在哪些事务上需求较高”、“处理哪里事务 上需要完善衔接过程” “哪些事务应当与信息化技 术结合”，因地制宜地完善 “一站式”服务中心人 员结构, 开展针对性技术技能培训, 如电脑操作培 训以及处理各类学生事务的信息化技术实际操作 培训, 坚持以 “学生事务需求” 为核心, 提升 “一 站式”服务中心人员综合能力。

\section{5. 结论}

高校 “一站式” 服务模式以 “服务” 为核心， 以 “需求” 为目标, 通过创新形式解决学生事务与 教职工事务,

其值得我们从多角度、多层次、多方面进行深刻反 思与探讨。

本文从 “学生事务” 视角反思与探讨高校 “一 站式” 服务模式，本文结构主要分为三部分：

第一、从国内高校 “一站式” 服务模式现状出 发, 将 “一站式” 服务模式特点归纳为服务性、专 业性、综合性、多样性、专业性。

第二、根据 “一站式” 服务模式特点分析出目 前 “一站式” 服务模式存在的现实困境: “一站式” 服务中心综合性止于表面、“一站式”服务中心信 息化技术水平较低、“一站式” 服务中心职工人员 综合能力较差, 职工人员结构有待优化。

第三、结合防疫控疫的具体要求，提出一站式” 服务中心优化途径，探讨符合现实需求的 “一站式” 服务模式: 促进 “一站式” 服务模式与 “互联网” 相结合、创新改革 “一站式” 服务中心组织结构、 调整 “一站式” 服务中心人员及优化职工技术技能
培训

最后，本文认为反思与探讨 “一站式” 服务模 式必须坚持与时俱进的理念, 要在社会变迁中创新 驱动 “一站式” 服务模式, 而非墨守成规地把 “一 站式”服务模式推向 “新的传统模式”。

\section{项目基金}

本文为广东海洋大学第七期学生工作 研究课题《广东海洋大学 “一站式” 学生 服务中心的可行性研究与建设》(gdou0718) 的阶段性成果。

\section{REFERENCES}

[1] Wang Zhanren. Philosophy and Practice of "One-stop Service" for Student Affairs in British Universities $[\mathrm{J}]$. Research on Ideological Education,2010(06):79-82.

[2] Liu Jun, Guo Jia, Mo Zi-Feng. Practice and exploration of "service education" in universities -- a case study of one-stop service hall of Guangdong university of foreign studies [J]. Journal of Inner Mongolia normal university (education science edition),2016,29(10):82-86.

[3] Zhang Ning. Discussion on one-stop Service Mode of College Student Affairs under the background of "Internet +" -- A Case study of Hebei University of Economics and Business [J]. Think Tank Era,2020(09):134-135.

[4] Wang Changheng. Research on college students' affairs management $[\mathrm{J}]$. Journal of Shandong university of technology (social science edition),2017,33(03):106-108.

[5] Yu Honghao. Research on the Development Status and Countermeasures of One-stop Service Centers in Colleges and Universities [J]. International Public Relations, 2020(08):214-215.

[6] Yan Bingming. Discussion on the Construction Practice of "One-stop" College Student Affairs Service Center [J]. Public Relations World,2020(02):147-148.

[7] Yang Mingzhu. Research on Ideological and Political Education Mode of College Students under one-stop Service of Student Work [J]. Quality Education in West China, 2012,4(22):47+49.

[8] Liu Xiangyu, Zou Yunlong, Xu Hongyan, Hou Yingzhe, Qi Jialiang. Practical Exploration of one-stop Service Mode of university Informatization under the 
Background of Internet $+[\mathrm{J}]$. Education Modernization, 2015,5(46):271-273.

[9] Chen Wenqian, Yan Zhongcheng. Research on the Construction of One-stop Administrative Service Center in Colleges and Universities [J]. China Modern Educational Equipment,2018(11):1-3. 